\title{
ENSEÑANZA VIRTUAL DE LENGUA INGLESA DURANTE EL CONFINAMIENTO DOMICILIARIO: PERCEPCIONES Y REACCIONES DEL ALUMNADO EN UNA UNIVERSIDAD ESPAÑOLA
}

\author{
Teaching English Online during the National Lockdown: Students' \\ Perceptions and Experiences at a Spanish University \\ ENSEIGNEMENT EN LIGNE DE LA LANGUE ANGLAISE PENDANT LE CONFINEMENT \\ DOMICILIAIRE : PERCEPTIONS ET RÉACTIONS DES ÉTUDIANTS DANS UNE UNIVERSITÉ \\ ESPAGNOLE
}

Bruno Echauri-Galván

Profesor ayudante doctor,

Universidad de Alcalá, Alcalá de

Henares, España.

bruno.echauri@uah.es

https://orcid.

org/0000-0002-7055-5699

\section{Silvia García-Hernández}

Profesora ayudante doctora,

Universidad de Alcalá, Alcalá de

Henares, España.

silvia.garciah@uah.es

https://orcid.

org/0000-0001-8276-604X

María Jesús Fernández-Gil

Profesora titular de universidad, Universidad de Alcalá, Alcalá de Henares, España.

mi.fernandezg@uah.es

https://orcid.

org/0000-0001-8813-6401

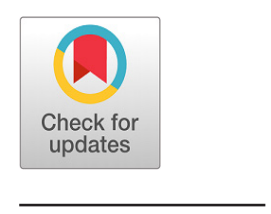

\section{RESUMEN}

La explosión de la pandemia de Covid-19 en España supuso un reto mayúsculo para las universidades con educación presencial ya que estas fueron obligadas a adaptarse a la docencia en línea de manera casi inmediata. Este artículo tiene el objetivo de ofrecer una instantánea de los resultados de este salto repentino a un modelo virtual en la enseñanza de lengua inglesa en distintos programas de grado de la Universidad de Alcalá en España. Para ello, se centra en las percepciones y reacciones del alumnado. Los datos derivaron de una encuesta respondida por 159 estudiantes de Inglés de las facultades de Filosofía y Letras; y de Ciencias Económicas, Empresariales y Turismo, y de un grupo de discusión posterior en el que participaron los autores del estudio y una selección de los encuestados. Estos dos métodos se utilizaron para ahondar en el tratamiento dado a aspectos centrales de la enseñanza de lenguas extranjeras, tales como: (a) la adquisición de competencias, (b) los métodos de evaluación, (c) la utilidad de las tecnologías de la información y la comunicación (TIC), y (d) la interacción entre estudiantes y entre estudiantes y docentes, durante el periodo de enseñanza a distancia que sobrevino a la pandemia. Los resultados evidenciaron la problemática que entraña esta modalidad virtual en cuanto a la adquisición y la práctica de destrezas como la expresión oral, la participación en clase, la articulación del trabajo en grupo y las relaciones interpersonales. Además, subrayaron beneficios, tales como un mayor conocimiento y manejo de los recursos tecnológicos. Finalmente, sirvieron para mostrar al profesorado los instrumentos digitales de trabajo y evaluación mejor y peor valorados por el estudiantado.

Palabras clave: Covid-19; enseñanza de lenguas extranjeras; enseñanza del inglés; enseñanza virtual; percepciones de los estudiantes; TIC.

Recibido : 2021-02-17 / Aceptado : 2021-06-30 / Publicado : 2021-09-11

https://doi.org/10.17533/udea.ikala.v26n3a08

Edición especial sobre The Role of Technology in Language Teaching and Learning amid the Crisis Generated by the COVID-19 Pandemic. Editores: Marta González-Lloret, University of Hawai'i at Mānoa, EE.uU.; Laia Canals, Universitat Oberta de Catalunya, España; Jorge Pineda, Universidad de Antioquia, Colombia.

(C) 2021 Universidad de Antioquia. Este es un artículo publicado en acceso libre, en los términos de la Licencia Creative Commons BY-NC-SA 4.0 Internacional. 


\begin{abstract}
The outbreak of the Covid-19 pandemic in Spain was a major challenge for universities offering face-to-face education since these were compelled to adjust to online teaching in an extremely brief time span. This article aims to offer a comprehensive picture of the repercussions that such a sudden immersion in full virtual mode had on instrumental English subjects taught in different undergraduate programs at Universidad de Alcalá in Spain. The profile is based on the data collected from: (a) a survey with 159 respondents enrolled in English courses in the faculties of Philosophy and Arts, and of Economics, Business and Tourism; and (b) a subsequent discussion group, including the authors of the study and a deliberately selected number of respondents. Both methods were used to delve into core aspects of language teaching such as: (a) skills acquisition, (b) assessment methods, (c) the usefulness of the ICT tools employed in the classroom, or in-class, and (d) interactions between students and between students and teachers in this exceptional context of unforeseen remote education. Results underscore the problems this scenario entails for the practice and acquisition of skills such as oral performance, class participation, or the management of teamwork and interpersonal relationships. They also pinpoint certain benefits related to an enhanced knowledge and handling of ICT tools. Finally, they give instructors insight into the digital applications and assessment instruments which were more and less highly valued by students.
\end{abstract}

Keywords: Covid-19; foreign language teaching; English teaching; online teaching; ICT; students' perceptions.

\title{
RÉSUMÉ
}

L'explosion de la pandémie de covid-19 en Espagne a constitué un défi majeur pour les universités qui offrent des cours présentiels, car elles ont été obligées à s'adapter à l'enseignement à distance d'une manière presque immédiate. Le présent article se concentre sur les expériences des étudiants et vise à offrir un instantané des résultats de ce saut soudain vers un modèle virtuel de l'enseignement de la langue anglaise dans les différentes licences de l'Université d'Alcalá, en Espagne. Les données sont issues d'une enquête répondue par 159 étudiants inscrits dans les cours d'anglais des facultés de Philosophie et Lettres, et de Sciences Économiques, de l'Entreprise et du Tourisme, ainsi comme d'un groupe de discussion postérieur, où les auteurs de cette étude et une sélection des interviewés ont participé. Ces deux instruments nous ont permis d'approfondir les questions essentielles de l'enseignement des langues, telles que a) l'acquisition des compétences, b) les méthodes d'évaluation, c) l'utilité des tecnologies de l'information et la communication (TIC), et d) l'interaction dans la classe entre les étudiants et entre l'enseignant et l'apprenant dans ce cadre exceptionnel de l'enseignement à distance. Les résultats ont mis en évidence les défis de cette modalité virtuelle concernant l'acquisition et la pratique de compétences, telles que l'expression orale ; la participation en cours ; l'articulation du travail de groupe et les relations interpersonnelles. De plus, ils ont souligné des bénéfices, tels qu'une meilleure connaissance et gestion des ressources technologiques, et ont donné un aperçu aux professeurs des outils numériques de travail et d'évaluation les plus et les moins appréciés par les étudiants.

Mots clés : Covid-19 ; acquisition d'une langue étrangère ; enseignement d'anglais; enseignement en ligne; TIC; perceptions des étudiants. 


\section{Introducción}

El confinamiento generalizado de la población, al que llevó la eclosión de la pandemia de covid- 19 en España en marzo de 2020, impactó sobre todos los niveles y programas educativos del país. La educación primaria, secundaria y superior pasaron a un modelo virtual de forma casi inmediata y los planes basados en la presencialidad tuvieron que ser repensados de manera urgente. Las ramificaciones pedagógicas de un cambio tan abrupto y de esta magnitud son innumerables y golpean aspectos formativos clave, como la organización de actividades y tareas, la adquisición de destrezas y conocimientos, los métodos de evaluación, las dinámicas de clase o las relaciones estudianteestudiante y estudiante-docente, entre muchos otros. Al mismo tiempo, el nuevo escenario exige una adaptación al medio que pasa, forzosamente, por un desarrollo exponencial de la competencia digital por parte del profesorado y los discentes.

El presente artículo muestra el impacto de todo lo anterior en un entorno concreto: el de la enseñanza de lengua inglesa en la Universidad de Alcalá (UAH), España, para lo cual responderá a los siguientes interrogantes:

1. ¿Hastaquépunto, según el estudiantado, hainfluido el salto virtual en su adquisición de destrezas?

2. ¿Cómo creen que afecta el entorno virtual a las dinámicasdetrabajoyalarelación docente-discente?

3. ¿Qué grado de utilidad han tenido las distintas herramientas informáticas utilizadas en el proceso de enseñanza-aprendizaje durante este periodo?

4. Entre los estudiantes, ¿cuál ha sido el nivel de aceptación de los métodos de evaluación aplicados por el profesorado?

A través de estas preguntas, esta investigación busca ofrecer una instantánea del efecto atribuible al salto repentino a un modelo de enseñanza virtual en el caso de una institución de enseñanza superior concebida como puramente presencial.
El estudio se construye sobre dos puntos axiales: una encuesta, en la que participan estudiantes de distintos grados que cursaron asignaturas de inglés instrumental durante el confinamiento, y un grupo de discusión posterior, en el que se profundizó en los aspectos más dilemáticos y opacos de los resultados.

Somos conscientes de que las respuestas y conclusiones que ofrecen las siguientes páginas surgen en un marco y momento muy específicos. Esto, lógicamente, limita su capacidad de extrapolación a otros contextos y su uso como herramientas universales. Pero no es nuestro objetivo aportar certezas absolutas a problemas complejos, sino trasladar una experiencia para que otros puedan conocerla, analizarla, debatirla y enriquecerla.

Artículos como este responden al llamamiento de Jandrić (2020) para focalizar esfuerzos sobre la miríada de consecuencias de la pandemia y emplear la investigación como instrumento multidisciplinar y comprehensivo de profundización, reflexión y cambio. Su voz apela a la responsabilidad de la Universidad para con el entorno y a multiplicar los acercamientos intelectuales a la (ir) realidad que ha creado la COVID-19 en cada esfera de nuestras vidas.

En resumen, se trata de construir, pieza a pieza, desde ópticas varias, una bibliografía amplia y sólida que nos permita comprender cada ángulo de lo ocurrido para, el día de mañana, enfrentar con más garantías escenarios idénticos, similares a esa realidad pospandémica, que acabará llegando tarde o temprano. Desde un pequeño rincón del ecosistema académico, este es nuestro granito de arena.

\section{Marco teórico}

La irrupción de la Covid-19 cambió todo en una ventana de tiempo casi imperceptible. La pandemia tensionó y sigue tensionando el tejido económico-social de muchos países y supuso una prueba de estrés para los Gobiernos de todo el mundo y los pilares sobre los que se sustentan. 
La educación es, sin duda, uno de esos pilares y también uno de los ámbitos cuyo orden se ha subvertido en mayor medida. Como muestra, baste decir que, a 31 de marzo de 2020, se contabilizaron en el mundo más de 1500 millones de estudiantes afectados por la suspensión de las sesiones presenciales y el salto a un modelo de enseñanza en línea (Unesco, 2020).

Este nuevo contexto educativo planteó una problemática generalizada, pero especialmente aguda para aquellas instituciones de naturaleza eminentemente presencial, cuyo grado de digitalización y preparación para entornos virtuales dista mucho del de los centros de enseñanza remota. Gran parte de la educación superior en España, un país donde solo 6 de 83 universidades ofertan su docencia completamente en línea (España, Ministerio de Universidades, 2020), se vio afectada por esta situación y obligada a ajustar el proceso de enseñanza-aprendizaje al contexto digital, a través de sus distintas herramientas y posibilidades. Con tiempo y medios limitados y unos docentes y discentes acostumbrados a otros métodos, el reto para la red universitaria española fue mayúsculo.

Aquellos que acepten como válido el mantra de que toda crisis es, al mismo tiempo, una oportunidad, convendrán en que la situación excepcional que se vivió durante el segundo cuatrimestre del curso 2019/2020 y las réplicas que aún nos acompañan a fecha de cierre de este artículo han servido para poner en primerísimo plano el papel de la tecnología en la enseñanza y los potenciales beneficios de una docencia más digitalizada. Las opiniones a favor de una mayor simbiosis entre pedagogía y avances técnicos no son, sin embargo, algo nuevo, sino una postura ya defendida en foros educativos desde hace tiempo.

\section{La tecnología en la enseñanza universitaria}

En las últimas décadas, la incorporación de la tecnología a la enseñanza ha estimulado la innovación en las prácticas docentes e impulsado una transformación de los modelos pedagógicos hacia unas metodologías más dinámicas y flexibles
(Blessinger y Wankel, 2013), apoyadas en una mayor variedad de recursos pedagógicos accesibles y adaptables al aula gracias a las tecnologías de la información y la comunicación (TIC).

Entre las ventajas que dimanan de estos avances destacan el acceso más rápido y directo a un amplio abanico de materiales, la posibilidad de combinar varios instrumentos multimedia en una misma acción docente o la dinamización del proceso de enseñanza-aprendizaje a partir de estrategias como la gamificación y el aula invertida (Durán Medina et al., 2018).

De igual manera, estas nuevas metodologías han dado paso a una enseñanza mucho más centrada en el estudiantado y su autonomía, aspectos íntimamente ligados a un aumento notable de la motivación. Varios son los estudios que han demostrado un incremento en la motivación del alumnado cuando se utilizan este tipo de estrategias basadas en las TIC (Macaro, 2003; Sánchez Calderón, 2020). El refuerzo de este parámetro genera, además, beneficios adicionales para los estudiantes, como una mejor concentración en la tarea, unos niveles de atención más altos y una mayor interacción con otros recursos disponibles y con sus compañeros en actividades grupales (Cacheiro González, 2018; Zheng et al., 2016).

Asimismo, las TIC facilitan la supervisión por parte del profesorado y la comunicación entre docentes y discentes, al tiempo que favorecen la atención a la diversidad. En relación con el primer punto, la tecnología abre nuevos espacios de contacto entre las partes involucradas en el proceso de enseñanza-aprendizaje y permite acortar el espacio transaccional entre ellas (Cenich y Santos, 2005). El correo electrónico, las videollamadas y videoconferencias, los foros, los chats y otras aplicaciones de mensajería instantánea permiten articular una interacción más continua y estrecha que puede aprovecharse de varias formas: monitorización, resolución de dudas, mayor colaboración en el desarrollo de la asignatura o aclaración de conceptos, entre otras. 
En lo que concierne a la atención a la diversidad, cabe señalar que los distintos tipos de actividades que pueden implementarse en las aulas y los recursos al alcance de discentes y docentes coadyuvan a adaptar los materiales educativos a las necesidades del estudiantado o a cualquier dificultad que este pudiera presentar. Dicho de otro modo, las TIC ayudan a crear y presentar materiales adecuados para toda clase de estudiantes de forma inmediata y más sencilla (Loveless, 2003; Shamir y Margalit, 2014).

\section{La enseñanza de lenguas extranjeras y las tecnologías}

En cuanto a la enseñanza de idiomas en la que se enmarca este artículo, varias voces y estudios han abordado en los últimos años las posibilidades que las herramientas informáticas ofrecen al área en cuestión. Dichas investigaciones son poliédricas y entroncan con distintos planos del complejo proceso de aprendizaje de una lengua extranjera (LE). Sin embargo, son muchas las que coinciden en la importancia de la integración de la tecnología en las aulas de LE, ya que, a diferencia de la enseñanza tradicional, la introducción de recursos multimedia enriquece la presentación de la lengua objeto de estudio e incrementa la retención del lenguaje (Yaverbaum et al., 1997).

Para ello, se aprovechan muchas de las ventajas generales de las TIC descritas en párrafos previos. Por ejemplo, la interpolación de recursos en línea en la clase de idiomas permite acceder con rapidez y facilidad a gran cantidad de materiales para practicar todas las destrezas de manera interactiva. Así mismo, los estudiantes pueden estar expuestos con inmediatez a una panoplia de textos válidos, escritos u orales, que les permiten comprobar el funcionamiento de la LE de una forma contextualizada y adaptada a sus intereses, convirtiendo de este modo el proceso de aprendizaje en una experiencia significativa y auténtica.

Otro de los puntos clave de la integración de las TIC en la enseñanza de LE es la posibilidad que ofrecen de proseguir el contacto con ellas fuera del aula, conectando así el uso real de la lengua con los conocimientos adquiridos en la clase (Al-Mahrooqi y Troudi, 2014). Utilizando cualquier dispositivo con acceso a internet, los estudiantes pueden aumentar el tiempo de exposición y la interacción con la segunda lengua (L2), lo que permite seguir aprendiendo fuera del contexto educativo y promueve una mayor autonomía en el aprendizaje (Yildiz, 2020).

Dicha continuidad igualmente ofrece al estudiantado la posibilidad de incorporar de manera automática el componente cultural, accediendo a materiales auténticos que van más allá de los recursos calificados como "artificiales", por haber sido específicamente creados para la enseñanza de ciertos aspectos de la lengua en cuestión. Asimismo, el uso de las TIC habilita la práctica sincrónica con hablantes nativos o con hablantes de otras lenguas que también están adquiriendo la misma LE por medio de programas de intercambio virtual, lo cual favorece el desarrollo de habilidades comunicativas interpersonales (Cacheiro González, 2018) y la comprensión intercultural (Marczak, 2013; O’Dowd y Dooly, 2020), incluyendo, además, la posibilidad de exponerse a un entorno más polifónico a través del contacto con variedades diatópicas.

Como se ha subrayado anteriormente, al limitar el papel central del profesor y multiplicar las vías de interacción con la LE, las TIC además hacen posible reducir los niveles de ansiedad a la hora de practicar una L2. Ciertas tareas interactivas, por ejemplo, permiten al alumnado trabajar la expresión oral de forma más natural, sin sentirse observado directamente por el profesorado, con lo que se reduce la ansiedad y el miedo a cometer errores que puede provocar tener que comunicarse en una lengua no materna delante de un grupo de estudiantes o docentes (Al-Mahrooqi y Troudi, 2014). Asimismo, actividades como grabar y escuchar sus interacciones facilitan al estudiantado identificar sus propios fallos (gramaticales, de pronunciación y entonación, o del uso apropiado 
del léxico, entre otros), lo cual ayuda a aumentar la precitada autonomía en la adquisición de conocimientos, desarrollar la destreza de aprender a aprender y sentirse protagonistas de su propio aprendizaje con actividades de autoevaluación.

Las reflexiones anteriores enlazan con la visión de la tecnología como herramienta de transformación, elemento democratizador y catalizadora de una realidad más interconectada, libre e inclusiva, que autores como McLaren et al. (2020) han defendido recientemente. Sin embargo, sería un error mitificar los horizontes abiertos por las TIC y perder el espíritu crítico que debe acompañar a todo viento de cambio.

La discusión debe ser siempre polifónica y, por ello, conviene traer a este foro voces que subrayen los peligros que plantea el uso de las tecnologías en la enseñanza en general y, por extensión, en la impartición de una lengua extranjera. En este sentido, autores como Ramón-Ruiz (2020) alertan sobre cómo la educación remota puede ahondar en las desigualdades educativas ya existentes. En su opinión, la consolidación de un entorno gradualmente más virtual podría abrir nuevas brechas a nivel de infraestructuras, conectividad y acceso a los recursos tecnológicos y formativos, que complicarían (aún más) el acceso equitativo a una educación que aspira a la igualdad de oportunidades profesionales.

Siguiendo esta línea, estudios empíricos como el de Rodicio-García et al. (2020) analizan la huella que los supuestos anteriores han dejado en España tras el periodo de formación a distancia impuesto por el confinamiento. Su trabajo, centrado en estudiantes de todos los niveles educativos a partir de la Educación Secundaria Obligatoria, muestra cómo las limitaciones de acceso a los recursos no son el único escollo que debe superar el alumnado, sino que son una pieza más engranada a otras muchas, como una competencia digital por desarrollar o las complicaciones a la hora de enfrentarse a un cambio de escenario tan súbito. Conviene subrayar que, además, las dificultades se agravan en localidades con una población inferior a 10000 habitantes, lo que amplía la dimensión de una problemática que no es solo económica, sino también geográfica.

Otras propuestas, como la de Almazán-Gómez (2020), enarbolan muchas de las banderas rojas anteriores, al tiempo que ponen el foco sobre un aspecto de la educación digitalizada que suele ocupar planos periféricos en los estudios pedagógicos: el entramado societario que la impulsa y se nutre de ella. Y es que, al margen de los desequilibrios que un entorno virtual puede abrir entre estudiantes de un mismo centro, la tecnologización del sistema educativo supone también el enriquecimiento de industrias y empresas de dudoso compromiso social.

Investigaciones como las de Cobo (2019) profundizan en esta visión de la digitalización como negocio, y señalan que el abuso de la tecnología puede tener, además, consecuencias nefastas sobre el tejido formativo, como un mayor porcentaje de fracaso y abandono escolar. Del mismo modo, una excesiva tecnologización del contexto de aprendizaje entraña el peligro de "industrializarlo", automatizándolo y asimilándolo a cadenas de producción en serie que, en palabras de AlmazánGómez (2020), acaban "reduciendo a los alumnos a puros números” (párr. 14).

Los ya citados y otros muchos trabajos nos advierten de que un uso masivo de las TIC tiene importantes ramificaciones dentro y más allá del aula universitaria, no todas positivas, por lo que conviene pensar muy bien la dirección de cada paso que demos en ese sentido. Es posible que la máxima recogida en párrafos anteriores sea cierta y que toda crisis suponga, en efecto, una oportunidad. Pero son también el ecosistema perfecto para abrir heridas profundas y cometer errores de los que arrepentirnos más tarde.

Los argumentos previos revelan lo dilemático de la situación, los desafíos que entraña el acelerado desarrollo tecnológico actual y, de forma 
más específica, los retos que plantea para la formación reglada, tanto a nivel de aula como a nivel institucional.

Ante un escenario tan incierto, en el que la crisis sanitaria augura una presencia cuando menos temporal y parcial de la educación a distancia, creemos imperativo conocer el punto de vista del estudiantado, sus experiencias y sus reacciones ante un entorno, el virtual, impuesto, pero que muchos consideran parte indispensable del futuro de la docencia universitaria. Sus opiniones en este sentido resultan especialmente valiosas para la práctica docente y para preparar mejor al profesorado y a las universidades ante el más que previsible asentamiento de una realidad formativa posdigital, en la que la tecnología y otras dinámicas más tradicionales deberían conformar un andamiaje consistente que redunde en beneficio del alumnado.

\section{Enseñanza virtual de Lengua Inglesa en la Universidad de Alcalá}

Si la problemática presentada hasta ahora resulta de por sí compleja, no podemos obviar el efecto multiplicador que generan las particularidades de cada región y cada centro educativo. En este sentido y de cara a comprender mejor el contexto y los resultados de este estudio, conviene desglosar algunos datos de interés sobre la institución en la que se llevó a cabo.

La UAH es un centro de educación superior eminentemente presencial y el uso de las herramientas informáticas puestas a disposición del profesorado o empleadas por estos últimos a título personal no tiene vocación sustitutiva, sino de refuerzo y ampliación de los conocimientos impartidos en el aula. La plataforma que centraliza los contenidos y las acciones virtuales, y a la que tanto estudiantes como profesores tienen acceso, es Blackboard ${ }^{\circledR}$.

En un contexto de docencia $100 \%$ remota, el uso de esta plataforma dejó de ser un complemento para pasar a ser, necesariamente, la columna vertebral de la enseñanza. Este cambio de escenario llevó al profesorado de la UAH en general, y del área de Filología Inglesa en particular, a profundizar en el uso de distintas herramientas disponibles en Blackboard ${ }^{\circledR}$, cuya naturaleza y funcionamiento conectan con muchas de las utilidades de las TIC descritas en la sección anterior: foros, chats $\mathrm{y}$ videoconferencias; sondeos y actividades en línea; grupos de trabajo; compartición y transferencia de archivos... Estos y otros instrumentos se convirtieron en puntos cardinales y asideros recurrentes, ya que fueron los mecanismos que permitieron al profesorado de inglés como LE transformar las aulas en pantallas primero y, posteriormente, repensar y confeccionar actividades para desarrollar las distintas destrezas, abrir nuevos espacios de comunicación, dar continuidad al contacto con la L2 y acceso a materiales relevantes, u organizar acciones docentes combinando varios recursos digitales.

Si bien el entorno virtual es un eje fundamental de este estudio, otros como el social y el geográfico tienen también su lógica incidencia. La UAH es una institución de carácter pública, en la que estudian aprendientes de muy distintos lugares y trasfondos sociales. Este es un detalle importante, puesto que las diferencias entre el tejido familiar y económico son dos de las principales causas de la llamada "brecha digital", según autores como Dey y Ali (2016) o Van Dijk (2017). En este sentido, las asimetrías en el acceso a recursos tecnológicos e informáticos y el desacompasamiento de los ritmos de aprendizaje que implican son peligros que sobrevuelan aquellas instituciones que, como la nuestra, acogen a estudiantes de perfiles socioeconómicos diferentes.

En lo que respecta a la lengua inglesa, su enseñanza atraviesa múltiples grados de naturaleza diversa. Por descontado, el inglés instrumental es una materia nuclear en programas como los de Estudios Ingleses y Lenguas Modernas y Traducción, donde, además, se profundiza en la lengua desde ángulos más específicos como el cultural, el literario o el traductológico. Sin embargo, esta asignatura también es parte del plan de estudios de grados 
como Turismo y Administración y Dirección de Empresas, Humanidades, Magisterio, Estudios Hispánicos o Derecho, entre otros.

Los siguientes apartados presentan la información recabada entre estudiantes de varios de estos grados, con el objetivo de cartografiar el impacto del salto virtual en la enseñanza de inglés en la $\mathrm{UAH}$ y responder a las preguntas planteadas en la introducción.

\section{Método}

Este estudio, de carácter mixto (cuantitativo y cualitativo), contó con la autorización del Departamento de Filología Moderna de la UAH.

\section{Recolección y análisis de los datos}

Para llevar a cabo el estudio, se utilizaron dos procedimientos metodológicos coalescentes de recogida de datos: un cuestionario en línea y un grupo de discusión virtual. Antes de lanzar el primero, se informó a los participantes sobre la confidencialidad en el tratamiento de sus respuestas y su utilización únicamente con fines de investigación.

El diseño de este cuestionario se realizó orientando las preguntas a pulsar la satisfacción del estudiantado tras el periodo de docencia virtual sobrevenida, y sus opiniones sobre aspectos generales de la enseñanza en línea y del uso de las posibilidades docentes y de evaluación que ofrece Blackboard.

Una vez elaborado, el cuestionario fue revisado por los integrantes del Grupo de Innovación Docente del Aula de Lenguas Extranjeras (iDEALE) de la $\mathrm{UAH}$, al que igualmente pertenecen los autores de este artículo. Además, para confirmar su validez, se llevó a cabo un pilotaje, utilizando la técnica del muestreo por conveniencia, con un grupo de estudiantes de la población objeto de estudio. Tras recibir sus respuestas, revisarlas y verificar aspectos relativos al buen funcionamiento y visionado de la plantilla, se introdujeron las modificaciones pertinentes. También se efectúo un análisis de fiabilidad en la escala de percepciones sobre todos los ítems de la escala del cuestionario, obteniendo un alfa de Cronbach que indicaba un alto nivel de consistencia interna $(\alpha=0,82)$. El envío de la versión final y la recopilación de respuestas tuvieron lugar en mayo de 2020.

Dichas respuestas se codificaron de forma numérica para su posterior análisis estadístico. El esquema de codificación se elaboró de manera que las puntuaciones más altas representaran niveles más altos, de acuerdo con la afirmación propuesta. Asimismo, no se introdujeron enunciados negativos en el cuestionario, por lo que todas las preguntas se codificaron siguiendo la misma regla.

El cuestionario se seccionó de la siguiente forma:

- Valoraciones generales: sección orientada a conocer la visión de los estudiantes respecto a asuntos generales, como el nivel de motivación propio y del profesorado, la comunicación con los docentes, ventajas e inconvenientes de las clases a distancia, y niveles de participación y aprovechamiento.

- Herramientas docentes: incluye preguntas sobre las herramientas disponibles en Blackboard para la docencia telemática. Se pidió a los participantes que valorasen su utilidad según su criterio en una escala Likert impar de 5 puntos (siendo 1 "Nada útil" y 5 "Muy útil").

- Métodos de evaluación: centrada en los métodos de evaluación en línea que ofrece Blackboard. Los encuestados valoraron, siguiendo la escala Likert anteriormente descrita, la adecuación de estos métodos para la evaluación del aprendizaje del inglés como LE en el entorno virtual.

- Preguntas sobre el nivel de satisfacción general con los resultados del aprendizaje durante el periodo de clases a distancia.

Tras el análisis de resultados, se ofreció a los participantes la posibilidad de unirse a un grupo de discusión, para profundizar en sus respuestas y 
entablar una conversación con estudiantes de otros grados sobre la enseñanza y la evaluación virtuales durante el periodo de confinamiento. Nuestro objetivo era analizar, con más detenimiento, algunas de las conclusiones que arrojaban los resultados del cuestionario.

La implementación de este segundo método de recogida de datos se llevó a cabo en una sesión telemática, tras obtener el consentimiento escrito de los estudiantes interesados en participar para ser grabados.

El encuentro virtual orbitó en torno a los siguientes grandes temas, ya fuera por la disparidad obtenida en las respuestas recibidas en el cuestionario o para ahondar en algunos aspectos clave de la enseñanza virtual en este contexto concreto:

- La aparente contradicción entre la gran utilidad que los estudiantes ven al uso de la tecnología y el malestar que les genera la excesiva dependencia que les produce.

- La dificultad a la hora de practicar la expresión oral en la modalidad en línea.

- La diversidad de opiniones sobre el entorno virtual como factor disuasorio para la participación en clase.

- El aprovechamiento del aprendizaje en la enseñanza remota.

- El trabajo en grupo.

- El escaso uso de las tutorías.

- Las herramientas de la plataforma Blackboard ${ }^{\circledR}$ y su utilidad.

- La valoración sobre los métodos de evaluación empleados.

\section{Población y muestra}

El cuestionario se distribuyó entre un total de 270 estudiantes, pertenecientes a 7 titulaciones de grado diferentes, en cursos en los que el inglés se estudia como lengua instrumental y en los que están implicadas dos facultades distintas: la de Filosofía y Letras, y la de Ciencias Económicas, Empresariales y Turismo.

El número de respuestas recibidas fue 159 (casi un $60 \%$ del total de posibles participantes) y su distribución por especialidades fue la siguiente: Lenguas Modernas y Traducción (51,57 \%, el $70,08 \%$ del total de estudiantes matriculados), Turismo/Turismo y Administración y Dirección de Empresas (17,61 \% / 52,83 \%), Estudios Ingleses (17,61 \% / 51,85\%), Estudios Hispánicos $(8,18 \%$ / $52 \%)$ y Humanidades/Humanidades y Magisterio (5,03\% / 38,09\%).

La sesión del grupo de discusión se realizó el 24 de junio de 2020, a través de Blackboard ${ }^{\circledR}$. El encuentro estuvo moderado por los autores de este artículo y en él intervinieron cinco estudiantes de distintos grados. Esta selección se hizo siguiendo criterios de representatividad, que permitiesen incluir participantes de diferentes titulaciones y cursos. Los únicos grados no representados (por falta de voluntarios) fueron el grado en Turismo/Turismo y Administración y Dirección de Empresas y el grado en Humanidades/Humanidades y Magisterio.

A pesar de la variabilidad en el perfil académico del alumnado, el objeto de estudio es comparable, ya que calibra el impacto de la docencia virtual sobrevenida en discentes de asignaturas de inglés con rasgos comunes: primer ciclo de estudios, obligatoriedad y una carga lectiva de 3-4 horas semanales.

Para analizar los datos de este grupo de discusión, se llevó a cabo un análisis cualitativo de contenido, segmentado en dos etapas: primero, se transcribió la totalidad del encuentro, y a continuación, se categorizaron los datos verbales obtenidos y se clasificaron, centrándonos en las variables y los aspectos clave mencionados en el subapartado anterior. Así, mediante la comparación y el contraste de las respuestas de los participantes, pudieron establecerse distintos patrones 
de respuesta que ayudaron a matizar y completar la información cuantitativa obtenida en los cuestionarios.

\section{Resultados y discusión}

Los siguientes subapartados recogen, desgranan y evalúan las respuestas a cada una de las preguntas planteadas en el cuestionario, utilizando una serie de figuras y tablas para mostrar los principales datos obtenidos. El análisis de la vertiente cuantitativa de estos resultados se interpola con los comentarios y opiniones del grupo de discusión en los puntos pertinentes.

\section{Motivación de estudiantes y profesores}

Uno de los aspectos más relevantes en este apartado es que los y las respondientes consideran que la motivación del profesorado, en comparación con la suya propia, es casi un punto superior. Así, mientras la media sitúa el nivel de motivación de estos últimos a mitad de escala $(3,025)$, la de los primeros, atendiendo de manera exclusiva a la percepción del estudiantado, está más cerca del "Bastante motivado" (3,716). Cabe señalar, además, que la asimetría y la curtosis para ambos ítems oscila, como se recoge en la Tabla 1, entre $-0,5$ y 0 , con lo que puede afirmarse que los datos se distribuyen normalmente.

\section{Ventajas e inconvenientes de la docencia remota}

En línea con lo anterior, una de las mayores desventajas de la docencia virtual, según el alumnado, es el hecho de que esta despierta menor nivel de motivación que las clases presenciales, algo que parece contradecir las afirmaciones de Macaro (2003) y Sánchez Calderón (2020) recogidas en el marco contextual, pero que matizaremos unas líneas más adelante.

Con el 41,72\% de las respuestas, la pérdida de motivación se sitúa solo por detrás de la excesiva dependencia de la tecnología que lleva aparejada esta modalidad educativa, que el 50,33\% de los encuestados señala como el principal escollo del modelo virtual, y de los problemas de concentración derivados de distracciones domésticas, cuestión que incomoda al 47,66\%.

Cabe destacar que dos de estos aspectos negativos, falta de motivación y de concentración, están íntimamente vinculados al proceso de aprendizaje y que, con posterioridad, el grupo de discusión estableció que la dependencia tecnológica generaba problemas adicionales en ese mismo sentido. Es, por ende, plausible pensar que la pérdida de motivación y concentración no se debe tanto al empleo de la tecnología en el aula como a su uso exclusivo a lo largo del curso.

Este grado de dependencia tecnológica plantea otras derivadas que merecen subrayarse. Tal y como se comentaba en secciones anteriores, uno de los peligros de la digitalización masiva de la enseñanza es la acentuación de asimetrías entre estudiantes, fundamentadas (aunque no solo) en las dificultades de conectividad y acceso a los recursos digitales. Este factor ha tenido una clara incidencia en este estudio, ya que las personas encuestadas señalaron que las dificultades técnicas recurrentes y la falta de medios tecnológicos adecuados incidieron en el normal desarrollo y práctica de destrezas, y en el

Tabla 1 Motivación de estudiantes y profesores

\begin{tabular}{lccccc}
\hline & N & Media & $\begin{array}{c}\text { Desviación } \\
\text { estándar }\end{array}$ & Asimetría & Curtosis \\
\hline $\begin{array}{l}\text { Motivación } \\
\text { estudiantado }\end{array}$ & 159 & 3,025 & 0,947 & $-0,321$ & $-0,304$ \\
$\begin{array}{l}\text { Motivación } \\
\text { profesorado }\end{array}$ & 159 & 3,716 & 1,007 & $-0,572$ & $-0,121$ \\
\hline
\end{tabular}


tiempo útil y el aprovechamiento académico de las sesiones remotas, algo que ha podido influir negativamente en la visión del contexto puramente virtual en el que se desarrolló la docencia.

En el lado positivo, existe un amplio consenso $(79,9 \%)$ a la hora de señalar la comodidad como la gran ventaja de esta modalidad de enseñanza. Este dato quizás guarde relación con el contexto sociocultural que define la docencia de la UAH, que no solo atrae a alumnado del Corredor del Henares, el área geográfica en la que se sitúan sus tres campus universitarios, sino procedente de toda la Comunidad de Madrid, así como de provincias colindantes y otras más alejadas; estos últimos probablemente valoraron positivamente poder seguir con sus estudios sin tener que trasladarse hasta Alcalá de Henares o Guadalajara. Se trata, no obstante, de una variable que habría que estudiar con más detenimiento, puesto que no se tuvo en consideración en este trabajo.

La segunda gran ventaja identificada por el estudiantado es que este periodo de docencia virtual sirvió como medio de evasión. Hay que pensar que los meses en que la Universidad permaneció cerrada (de mayo a junio de 2020), corresponden al periodo de confinamiento duro, cuando en España se produjo un mayor número de fallecimientos por la pandemia. Parece razonable pensar que el alumnado encontró, en la posibilidad de continuar con sus estudios, una forma de mantenerse al margen de la crudeza de los datos.

Por otra parte, durante la discusión en grupo afloró una aparente contradicción respecto a los datos obtenidos en esta sección del cuestionario. $\mathrm{Y}$ es que si bien estos últimos apuntaban a que la dependencia tecnológica en la enseñanza virtual dificulta el aprendizaje, los integrantes del grupo definieron las clases como "provechosas". Esta opinión, subrayaron, se fundamenta en la metodología por medio de la cual se dio acceso a la materia y en el hecho de que parte del profesorado ofreciese correcciones personalizadas, dejando en segundo plano el grado de tecnologización del entorno de aprendizaje.

\section{Interacción profesores-estudiantes}

Los datos concernientes a la valoración sobre las relaciones docente-discente permiten afirmar que la falta de contacto directo durante el periodo estudiado no afectó la interacción entre las dos partes del proceso de enseñanza-aprendizaje. La distribución de los datos, cuya asimetría tiende a 0 $(-0,108)$ y que tiene un coeficiente negativo de curtosis $(-0,825)$, indica que la dispersión es muy similar a ambos lados de la media y que las colas son livianas (véase Tabla 2). De forma más específica, el 30,67\% de la muestra ha permanecido neutral respecto a si durante el periodo de clases a distancia se sintió más o menos cómodo trasladando dudas y dificultades a sus profesores, pues eligió el ítem a mitad de escala.

Por otro lado, la diferencia entre quienes dicen estar "Poco de acuerdo" y "Bastante de acuerdo" con la afirmación "Tengo menos reparo a la hora de hacerle llegar mis dudas y dificultades al profesor o a la profesora que si la clase fuera presencial" es de algo menos del $5 \%$, a favor de los que afirman haberse sentido más cómodos que en las clases presenciales. Esa tendencia positiva se revierte en los extremos de la escala, donde el $16 \%$ marcó el ítem "Nada de acuerdo", frente al $6 \%$ que marcó el ítem "Totalmente de acuerdo". En resumen, puede concluirse que el alumnado no refiere

Tabla 2 Interacción profesores-estudiantes

\begin{tabular}{lccccc}
\hline & N & Media & $\begin{array}{c}\text { Desviación } \\
\text { estándar }\end{array}$ & Asimetría & Curtosis \\
\hline $\begin{array}{l}\text { Contacto con el } \\
\text { profesorado }\end{array}$ & 159 & 2,874 & 1,145 & $-0,108$ & $-0,825$ \\
\hline
\end{tabular}


cambios sustanciales en lo que atañe al contacto con los profesores.

La conjunción de estas cifras con las obtenidas en el análisis de la motivación arroja un dato no menor, en el que conviene detenerse. Que los discentes sientan que el profesorado sigue realmente involucrado en su educación a pesar del cambio de medio y que su relación con ellos y ellas no se haya visto alterada a lo largo del curso, prueba que los peligros señalados por autores como AlmazánGómez (2020), con respecto a una automatización de la enseñanza a través de su tecnologización, son una advertencia y no una profecía que no podamos sortear. Es cierto que los datos aquí recabados se circunscriben a un contexto muy específico, pero también demuestran que la digitalización de un entorno concreto puede hacerse sin perder (al menos, no del todo) el factor humano, y sin la necesidad de anonimizar o cosificar la figura de estudiante.

\section{4}

En cuanto a los medios que emplearon para hacer efectivo el contacto con el profesorado, vemos que la comunicación tuvo lugar básicamente mediante dos canales: el correo electrónico y las clases por videoconferencia. Si bien uno y otro establecen una comunicación de distinto tipo (sincrónica y asincrónica, respectivamente), ambos fueron utilizados en similar medida (88,08 \% el primero, frente a $87,42 \%$ el segundo), lo que sugiere que el alumnado no necesita que la comunicación con el profesor fuera del horario lectivo dependa de un canal visual.

Sorprende comprobar que, a pesar de las múltiples formas de comunicación que ofrecen la plataforma Blackboard ${ }^{\ominus}$ y las Tic en su conjunto (foros, chats, mensajería, áreas de trabajo compartidas), únicamente son dos los medios escogidos para interactuar con el profesorado. En este mismo sentido, llama también la atención el hecho de que, pese a los numerosos avances y opciones disponibles, uno de estos medios sea, probablemente, el más tradicional de todos.
Asimismo, destaca que solo el 17,22 \% afirme haber solicitado una tutoría por videollamada, lo que confirmaría que el estudiantado no considera que la imagen y la inmediatez enriquezcan la comunicación con el personal docente, y eso a pesar de que la pandemia de Covid-19 ha disparado el uso de los servicios de videollamada y videoconferencia. En esta misma línea, la discusión en grupo reveló que muchas veces se recurrió a los últimos minutos de las clases para solicitar la aclaración de conceptos y dudas; de ahí que el alumnado no necesitase tutorías personalizadas, medio que, como señalaron ellos mismos y pueden atestiguar los discentes de los distintos grupos encuestados, tampoco es el preferido para contactar con el profesorado en la modalidad presencial.

\section{Dinámicas de trabajo}

Las respuestas tipo Likert revelan que el estudiantado prefirió las dinámicas de trabajo individual, porque le permitían organizar mejor su tiempo. Así, frente al 15,7 \% que no vincula esta modalidad de trabajo a una mayor eficiencia, el 52,2 \% de los respondientes la asocian a un mejor aprovechamiento del tiempo. Hubo, además, el $32 \%$ de respuestas en el punto medio, lo que implica que pocos encuestados ven el trabajo colaborativo como alternativa para ahorrar tiempo. La Figura 1 desglosa estos datos.

Paradójicamente, el número de participantes que afirmó "Echar de menos la interacción con los/as compañeros/as y poder seguir realizando

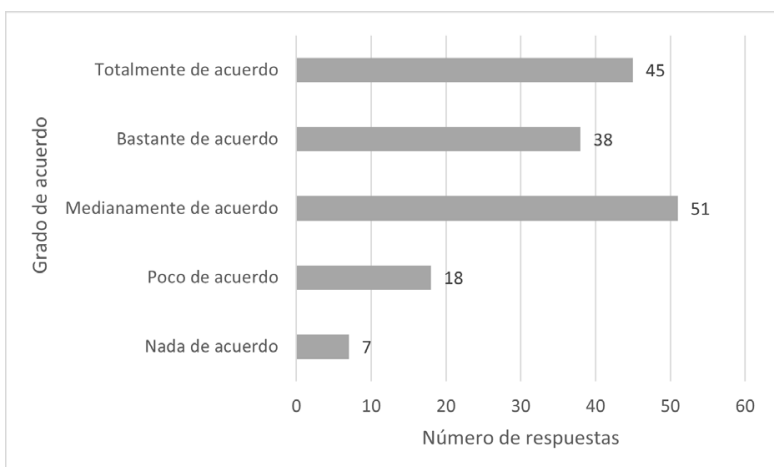

Figura 1 Trabajo individual y mejor gestión del tiempo 
actividades en pareja/grupos" es similar al de aquellos que valoraron el trabajo individual como una herramienta eficaz de gestión del tiempo, con una variación de tan solo 3 respuestas. De hecho, la diferencia entre medias asciende solo a 0,13 puntos, como muestra la Tabla 3.

Tabla 3 Trabajo individual y relaciones interpersonales en el aula

\begin{tabular}{lccc}
\hline & N & Media & $\begin{array}{c}\text { Desviación } \\
\text { estándar }\end{array}$ \\
\hline $\begin{array}{l}\text { Trabajo individual porque } \\
\text { organizo mejor el tiempo }\end{array}$ & 159 & 3,603 & 1,141 \\
$\begin{array}{l}\text { Echo de menos la } \\
\text { interacción }\end{array}$ & 159 & 3,465 & 1,189 \\
\hline
\end{tabular}

El grupo de discusión ayudó a matizar estos datos y clarificar esa aparente contradicción. Los participantes especificaron que la modalidad en línea dificulta la articulación de actividades grupales. Entre los motivos esgrimidos se cuentan los siguientes: los problemas de conexión, la aleatoriedad en la asignación de los subgrupos a través de la herramienta que ofrece Blackboard ${ }^{\circledR}$ y el impacto que tuvo el confinamiento en las rutinas diarias y, por tanto, en la posibilidad de adaptarse a los horarios de cada uno de los integrantes de un grupo de trabajo. También adujeron razones relacionadas con una carga de trabajo desequilibrada y con el estilo de aprendizaje de cada estudiante y su interés por la asignatura.

Las cuestiones apuntadas son de tipo operativo, con lo que puede concluirse que la paradoja es solo aparente. $\mathrm{Y}$ es que si el problema asociado con el trabajo en grupo reside en que este dificulta la gestión del tiempo, esto no invalida que se eche de menos el contacto personal y la interacción, que son propias de la modalidad de trabajo colaborativo.

De forma similar, la enseñanza virtual que recibió el alumnado también parece ir en detrimento de la participación en clase y del aprovechamiento de la asignatura. Aun conscientes de que se trata de dos aspectos de difícil evaluación por su intrínseco carácter cualitativo, resulta significativo que el 72,85\% considere que esta modalidad de enseñanza jugó en su contra en ambos planos (véase Figura 2). Los motivos, en este caso, no se atribuyen a los requisitos técnicos de los que depende la docencia en línea, sino a la distancia que media entre docente y discente, y a que esa falta de contacto favorece que no se siga el ritmo de la clase. Aunque no se verbalizara, este dato deja entrever la importancia de la interacción que se produce en la clase presencial con el profesorado y el hecho de que los múltiples canales de comunicación que ofrecen los entornos virtuales no parecen ser sustitutivo suficiente del trato cara a cara.

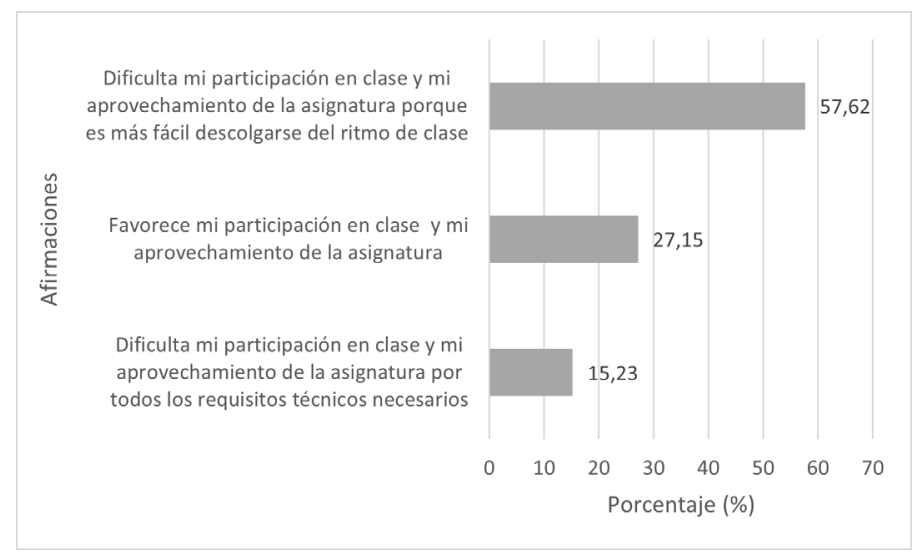

Figura 2 Impacto de la docencia virtual sobre la participación en clase y el aprovechamiento de la asignatura 


\section{Utilidad de las herramientas que ofrece Blackboard ${ }^{\oplus}$}

Como ya se ha comentado con anterioridad, las TIC, en general, y la plataforma Blackboard ${ }^{\circledR}$, en particular, amplían las vías de interacción y el abanico de actividades y recursos de los que docentes y discentes pueden hacer uso.

Los resultados obtenidos en relación con las herramientas de Blackboard ${ }^{\circledR}$ utilizadas durante el periodo de docencia $100 \%$ virtual revelan que las mejor valoradas son las siguientes: la sala de videoconferencia y chat $(88 \%)$, los materiales de apoyo a la docencia $(54 \%)$ y las actividades evaluables en línea $(52,67 \%)$ (véase Figura 3).

$\mathrm{Si}$ tomamos en consideración que estas tres herramientas facilitan la interacción dentro del aula y dejan fuera aspectos como la evaluación, los resultados de la sección entroncan con el precitado deseo de relacionarse con sus pares y permiten reforzar la conclusión de que, durante este periodo de enseñanza virtual, el estudiantado echó en falta la formación que tiene lugar en el espacio físico del aula.

Ello explica que el chat, que fortalece los lazos interpersonales, sea una de las herramientas mejor valoradas. Junto a esta aplicación destaca también la opción para compartir documentos y compartir pantalla/pizarra. En los tres casos, la media alcanza los 4,3 puntos.

Conviene, además, señalar que el número de participantes que se pronunció sobre la utilidad de los sondeos, la aplicación peor valorada, fue sensiblemente menor al que ofreció su opinión respecto de las otras utilidades de Blackboard ${ }^{\circledR}$. En una línea similar, la herramienta de grupos de trabajo, que permite formar equipos de aprendizaje en las sesiones virtuales, fue mayoritariamente calificada de "Poco" o "Nada útil", y definida en el grupo de discusión como una forma de trabajo que no acaba de convencer al estudiantado.

Existen dos factores que pueden explicar, siquiera parcialmente, las dos últimas reacciones. Por un lado, hay que pensar que el salto a la virtualidad fue tan brusco, que parte del profesorado, sin tiempo para renovar su modelo docente ni hacer ajustes en los objetivos y contenidos, se limitó muy posiblemente a transferir al espacio virtual los métodos, las técnicas y las estrategias de los que se valían en sus clases presenciales. Por otro lado, el paso a la enseñanza telemática en la UAH chocó con la limitada familiaridad del profesorado con este modelo. En un entorno de aprendizaje tradicionalmente presencial, el uso de Blackboard ${ }^{\bullet}$ y sus posibilidades suele ser accesorio y puntual, y la destreza en su manejo no es, en términos generales, la que demanda la docencia puramente telemática.

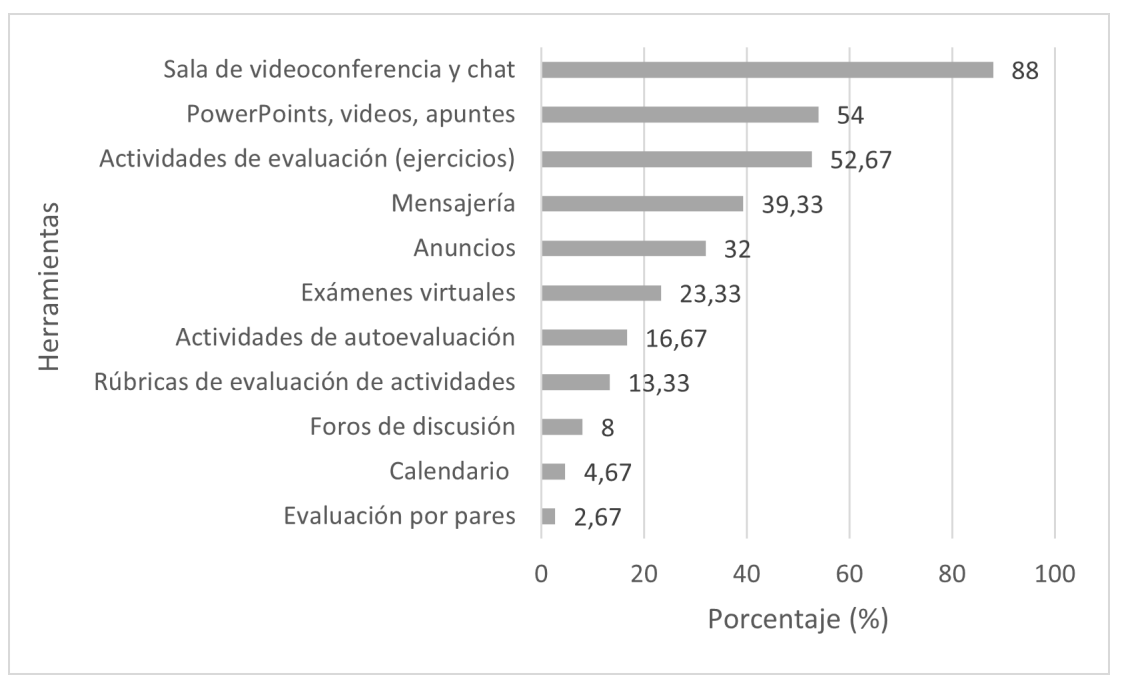

Figura 3 Utilidad de las herramientas que ofrece Blackboard ${ }^{\circ}$ 
Dentro de un escenario como este, con escaso margen para una correcta aclimatación y con una formación adicional que comenzó a recibirse ya avanzado el cuatrimestre, resulta complicado explotar, en su totalidad, las funcionalidades del entorno virtual de aprendizaje, y es muy probable que esto impidiese sacar un mayor partido a herramientas como los sondeos o los equipos de trabajo, potencialmente muy útiles, pero que requieren un conocimiento al menos intermedio de la plataforma.

Tal y como recoge el marco teórico, las TIC permiten la práctica de todas las destrezas relacionadas con el aprendizaje de una lengua. Eso no implica, sin embargo, que todas puedan o vayan a adquirirse con la misma facilidad y fluidez.

Preguntados por las dos destrezas más y menos difíciles de aprender en la modalidad de enseñanza virtual, el estudiantado mostró relativo consenso a la hora de señalar que la más fácil es la comprensión lectora $(48,34 \%)$, seguida de la expresión escrita (39,07\%). Ambas tienen en común que su práctica no está necesariamente condicionada por la interacción, lo que facilita el aprendizaje autónomo.

Por el contrario, las dos destrezas que resultan más complicado practicar desde casa son la comprensión oral $(39 ., 74 \%)$ y la expresión oral $(69,64 \%)$. En este último caso, si exceptuamos la práctica del monólogo, nos encontramos ante una destreza muy dependiente de dos partes que interactúen entre sí. En ese sentido y en especial si pensamos que la tecnología disponible hasta el momento limita la espontaneidad de la comunicación presencial, parece lógico que se identifique como un aspecto problemático. El grupo de discusión confirmó este punto, que puede explicar también la prevalencia de la práctica oral a partir de grabaciones y presentaciones sobre el ejercicio del diálogo sincrónico.

\section{Métodos de evaluación}

De entre los métodos de evaluación que ofrece Blackboard ${ }^{\oplus}$ hay un favorito evidente: las actividades de expresión escrita redactadas en casa. Esto se desprende de la media que obtiene este ítem, 4038 puntos, como de la asimetría, que tiene un claro sesgo hacia la derecha.

También concitan gran aceptación los ejercicios tipo test, las actividades de expresión oral pregrabadas y los resúmenes y comentarios de texto, todos con medias de 3,8.

Nótese, además, que los dos primeros métodos de evaluación muestran una distribución en la que, al igual que ocurre con los ejercicios de expresión escrita preparados con antelación, la cola se alarga para valores menores a la media, siendo la asimetría para los ejercicios de tipo test de $-0,629$, y para las actividades de expresión oral grabadas en casa, de $-0,777$.

Además de los datos aquí resaltados, la Tabla 4 recoge la media, la desviación estándar, la asimetría y la curtosis referentes a otros métodos de evaluación empleados en el aula de inglés como LE.

Tabla 4 Métodos de evaluación empleados en el aula de inglés como lengua extranjera

\begin{tabular}{lccccc}
\hline \multicolumn{1}{c}{ Métodos } & N & Media & $\begin{array}{c}\text { Desviación } \\
\text { estándar }\end{array}$ & Asimetría & Curtosis \\
\hline Foros de discusión/Wikis/Blogs & 100 & 3,376 & 1,028 & $-0,25$ & $-0,732$ \\
Expresión escrita en casa & 154 & 4,038 & 0,884 & $-0,764$ & $-0,527$ \\
Expresión oral grabada en casa & 145 & 3,868 & 1,042 & $-0,777$ & $-0,057$ \\
Ejercicios tipo test & 131 & 3,885 & 0,973 & $-0,629$ & $-0,086$ \\
Presentaciones orales & 143 & 3,363 & 1,110 & $-0,448$ & $-0,351$ \\
Ejercicios comprensión oral (Listening) & 136 & 3,588 & 1,043 & $-0,496$ & $-0,236$ \\
Resumen/comentario de texto & 149 & 3,859 & 0,870 & $-0,345$ & $-0,276$ \\
Portafolios digitales & 77 & 3,354 & 1,109 & $-0,458$ & $-0,126$ \\
\hline
\end{tabular}


Los datos obtenidos invitan a colegir que el estudiantado considera que la evaluación del desempeño académico en las asignaturas de inglés instrumental durante el periodo de docencia virtual sobrevenida debió apoyarse en herramientas que miden la ejecución individual, que permiten la realización de actividades asincrónicas y que dejan, por tanto, espacio a cierto grado de preparación.

En relación con esto, un aspecto abordado en el grupo de discusión es que las presentaciones orales en tiempo real no son adecuadas, porque dependen en exceso de la tecnología y generan ansiedad para quienes son tímidos, si bien esta última cuestión no es exclusiva del medio. Ello explica que este método de evaluación, sobre el que opinaron 143 encuestados, tenga una de las medias más bajas: 3363 .

Los participantes en el grupo de discusión igualmente mostraron escepticismo sobre la idoneidad de este método, que critican porque es una fuente de nervios. Si a este dato sumamos la antedicha preferencia por las actividades pregrabadas, observamos cómo las opiniones del estudiantado confluyen con el papel central que Al-Mahrooqi y Troudi (2014) dan a la ansiedad, y con su defensa del vídeo y las grabaciones como alternativa, siquiera puntual, al discurso sincrónico.

En lo tocante a los Foros de discusión/Wikis/ Blogs y los Portfolios digitales, la media obtenida permite concluir que también se consideran poco apropiados para la evaluación. El factor diferencial respecto a las presentaciones orales es que esta valoración responde a una opinión no informada del alumnado, en tanto son dos herramientas que reconocen no haber utilizado en la clase de Inglés a distancia. Este dato corrobora, en cierto modo, la precitada infrautilización por parte del profesorado de algunas posibilidades de la plataforma. Así, más de un tercio de los estudiantes (36,67\%) afirma no haber tenido oportunidad de utilizar los Foros de discusión/Wikis/Blogs, una cifra que se dispara en el caso de los Portfolios digitales $(51,33 \%)$.
Por otra parte, conviene apuntar que, cuando se trata de ponderar los métodos de evaluación, las medidas de asimetría y curtosis no indican una desviación anormal de la media, lo que apunta a un consenso entre los alumnos con relación a este aspecto de la docencia, con independencia de su estilo de aprendizaje.

Ese consenso es aún más evidente a la hora de señalar el sistema de evaluación que mejor se ajusta al aprendizaje del inglés como LE en la clase virtual. El 82,12 \% de las respuestas se inclina por la evaluación continua, vehiculada a través de actividades realizadas en clase y en casa. El examen en línea suscita un rechazo generalizado y solo el 3,31 \% lo considera la opción más adecuada de evaluación, una cuestión que se reafirmó en el debate mantenido en el grupo de discusión. En ese foro se adujeron las razones principales por las que este método no convence como mecanismo de evaluación: los exámenes se configuraron de forma que no era posible revisar las respuestas una vez completadas las distintas partes del ejercicio; no se dieron instrucciones claras sobre las especificidades de este tipo de examen, y no se dispuso de suficiente tiempo para completar las pruebas.

\section{Satisfacción general}

La distribución muestral de los datos que miden la satisfacción general del estudiantado con su rendimiento durante el periodo de clases a distancia apunta a que están contentos con la formación y la atención recibidas (véase Tabla 5). De hecho, el número de quienes dicen estar "Bastante satisfechos" ( 46 respondientes) es sensiblemente mayor al de quienes se declaran "Poco satisfechos" (26). Lo mismo sucede en los extremos de la escala, donde los "Muy satisfechos" (17 respondientes) superan a los "Nada satisfechos" (7). Además, el hecho de que el valor de la asimetría se acerque a $0(-0,202)$ y que la curtosis se sitúe en $\pm 0,5(-0,382)$, indica que los datos se distribuyen normalmente.

El grupo de discusión sirvió para reforzar la validez de esta interpretación, en tanto indicó estar satisfecho con la docencia en línea y haberse sentido 
Tabla 5 Satisfacción general

\begin{tabular}{llllll}
\hline & N & Media & $\begin{array}{c}\text { Desviación } \\
\text { estándar }\end{array}$ & Asimetría & Curtosis \\
\hline $\begin{array}{l}\text { Satisfacción } \\
\text { general }\end{array}$ & 159 & 3,289 & 1,014 & $-0,202$ & $-0,382$ \\
\hline
\end{tabular}

apoyado por el profesorado. En concreto, el grupo valoró positivamente la disponibilidad de estos últimos, los conocimientos tecnológicos adquiridos y el uso de una mayor variedad de recursos interactivos, aunque asimismo subrayó aspectos que podrían ser repensados en el futuro, especialmente la carga de trabajo y la evaluación.

\section{Conclusiones}

Como se señalaba anteriormente, la digitalización de la enseñanza es un proceso con anverso y reverso, al que conviene acercarse con espíritu crítico, más si cabe tras su aceleración a raíz de la pandemia que todavía sufrimos. Observar el fenómeno a través de esta lente solo es posible si se cuenta con una bibliografía amplia y nutrida, que explore sus aristas desde ángulos que engloben enfoques integrales, pero también de carácter local.

Es en esta línea donde se enmarca el estudio que aquí concluye y que ha pretendido pulsar las experiencias de los estudiantes de inglés como LE de la UAH durante el periodo de docencia en línea sobrevenida, provocado por la pandemia de Covid-19 y el cierre de los centros universitarios españoles en marzo de 2020. Con ello se buscaba identificar las principales dificultades a las que han tenido que hacer frente los estudiantes al pasar de un modelo puramente presencial a uno virtual.

Cabe destacar que, en general, la valoración es positiva. Así lo avala su grado de motivación, alto, pese a considerarlo inferior al alcanzado en contextos presenciales, y, en especial, su percepción de la motivación del profesorado, que juzgan superior a la propia.
Otro dato que apuntala lo anterior lo encontramos en la propia tecnología de la que depende esta modalidad docente. $\mathrm{Y}$ es que tanto la encuesta como la discusión en grupo revelan que el estudiantado aprecia la mayor variedad de recursos que se emplean en este tipo de enseñanza, así como los conocimientos tecnológicos adquiridos como consecuencia de desarrollar el proceso de enseñanza-aprendizaje en entornos digitalizados.

Esta visión positiva del entorno digital tiene, sin embargo, un envés que conviene no olvidar: gran parte de la muestra analizada ve como un problema la excesiva dependencia tecnológica. Esto nos remite a una cuestión subyacente a varias partes del artículo: la dificultad de encontrar ese equilibrio que nos permita implementar la tecnología de modo tal que potenciemos sus beneficios y limitemos sus desventajas.

En cuanto a las herramientas tecnológicas empleadas para la enseñanza y el aprendizaje del inglés como LE, las preguntas dirigidas a conocer las preferencias del alumnado ilustran que, aunque el abanico de recursos del que está dotada Blackboard ${ }^{\oplus}$ es amplio y todos se usaron en alguna medida, hay tres herramientas (Sala de videoconferencia y chat; PowerPoints, vídeos, enlaces y apuntes; y Actividades de evaluación en línea) que parecen monopolizar la práctica docente. Otros, por el contrario, como es el caso de los sondeos o las rúbricas de evaluación de actividades, no fueron explotados en toda su dimensión. Si bien creemos que son herramientas potencialmente útiles para la docencia virtual, hemos de tener en cuenta la excepcionalidad de las circunstancias en las que tuvo lugar la enseñanza en línea en este caso.

\section{9}


El profesorado, cuya docencia se enmarca en una universidad de naturaleza presencial, hubo de adaptar su metodología de manera súbita y hacer de un medio hasta entonces periférico la única vía para materializar el proceso de enseñanzaaprendizaje, lo que explica que no contase con el tiempo suficiente para explorar en profundidad sus posibilidades y que recurriese, por lo general, a usar las herramientas que reproducen las interacciones que tienen lugar dentro del aula.

Conscientes de que la realidad se impone y de que, tras la pandemia, es posible que la docencia bimodal gane peso en el engranaje universitario, desde la propia UAH se está trabajando para formar a sus profesores y con ello reducir ciertos problemas detectados por el alumnado en escenarios similares. A la luz de los resultados obtenidos en este estudio, esta formación parece que debiera dirigirse, por un lado, a mejorar las competencias digitales de los docentes, de modo que puedan sacar el máximo provecho de las herramientas puestas a su disposición; y, por otro, a ajustar los métodos de enseñanza y evaluación a las particularidades de esta modalidad. El objetivo último ha de ser crear una experiencia más positiva y fructífera, que permita reforzar los lazos socioafectivos en un entorno que tiende a describirse como frío y distante, y en el que la adquisición de ciertas competencias parece verse perjudicada.

Pasado un año del confinamiento domiciliario en España, creemos firmemente en el valor de seguir impulsando estudios que, como este, se nutren de una experiencia compartida con la que miles de docentes y discentes de todo el mundo pueden sentirse identificados de un modo u otro. Como en un laberinto de espejos, cada lector encontrará ante sí un reflejo distinto de su propia realidad, simétrico, parcial, combado o difuso, pero esperamos que esa comunión, ese reconocimiento, a pesar de las diferencias, pueda conducir a la reflexión y a la acción correlativa. Una acción que amplíe y enriquezca el espacio de debate, que nos ayude a comprender lo ocurrido hace unos meses, qué hicimos bien, en qué nos equivocamos, cómo seguimos adelante. No nos atrevemos a afirmar si las crisis son una oportunidad, una catástrofe o ambas cosas al mismo tiempo. Pero siempre deberían ser un aprendizaje.

\section{Referencias}

Al-Mahrooqi, R. y Troudi, S. (2014). Using technology in foreign language teaching. Cambridge Scholars Publishing.

Almazán-Gómez, A. (2020). covid-19: ¿punto sin retorno de la digitalización de la educación? Revista Internacional de Educación para la Justicia Social, 9(3), 1-4. https://revistas.uam.es/riejs/article/ view/12089/12009

Blessinger, P. y Wankel, C. (2013). Novel approaches in higher education: An introduction to web 2.0 and blended learning technologies. En C. Wankel y P. Blessinger (Eds.), Increasing students' engagement and retention in e-learning environments: Web 2.0 and blended learning technologies (pp. 3-16). Emerald.

Cacheiro González, M. L. (2018). Educación y tecnologia: estrategias didácticas para la integración de las TIC. UNED Publicaciones.

Cenich, G. y Santos, G. (2005). Propuesta de aprendizaje basado en proyecto y trabajo colaborativo: experiencia de un curso en línea. Revista Electrónica de Investigación Educativa, 7(2), 1-18. https://redie. uabc.mx/redie/article/view/133

Cobo, C. (2019). Acepto las condiciones. Usos y abusos de las tecnologias digitales. Santillana.

Dey, B. y Ali, F. (2016). A critical review of the ICT for development research. En B. Dey, K. Sorour y R. Filieri (Eds.), ICTs in developing countries (pp. 7-23). Palgrave Macmillan.

Durán Medina, J. F., Godoy Martín, F. J. y Martínez Terceño, J. (2018). Las TIC en las aulas de enseñanza superior. Gedisa.

España, Ministerio de Universidades (2020). Datos y cifras del sistema universitario español. https://www.educacionyfp.gob.es/dam/ jcr:b9e82c7a-1174-45ab-8191-c8b7e626f5aa/ informe-datos-y-cifras-del-sistema-universitarioespa-ol-2019-2020-corregido.pdf

Jandrić, P. (2020). Postdigital research in the time of covID-19. Postdigital Science and Education, 2, 233-238. https://doi.org/10.1007/s42438-020-00113-8 
Loveless, A. (2003). The role of ICT. Continuum.

Macaro, E. (2003). Teaching and learning a second language. A guide to recent research and its application. Continuum.

Marczak, M. (2013). Communication and information technology in (intercultural) language teaching. Cambridge Scholars Publishing.

McLaren, P., Wang, Y. y Jandrić, P. (2020). Reflections I and II: Reflections from education and the arts in the COVID-19 era. Communiars. Revista de Imagen, Artes y Educación Critica y Social, (4), 10-13. https:// revistascientificas.us.es/index.php/Communiars/ article/view/12779

O’Dowd, R. y Dooly, M. (2020). Intercultural communicative competence development through telecollaboration and virtual exchange. En J. Jane (Ed.), The Routledge handbook of language and intercultural communication (pp. 361-375). Routledge.

Ramón-Ruiz, G. (2020). Marcas de la pandemia: el derecho a la educación afectado. Revista Internacional de Educación para la Justicia Social, 9(3), 45-59. https://doi.org/10.15366/riejs2020.9.3.003

Rodicio-García, M. L., Ríos-de-Deus, M. P., MosqueraGonzález, M. J. y Penado Abilleira, M. (2020). La brecha digital en estudiantes españoles ante la crisis de la COVID-19. Revista Internacional de Educación para la Justicia Social, 9(3), 103-125. https://doi. org/10.15366/riejs2020.9.3.006
Sánchez Calderón, S. (2020). Learning English through ICT tools. Wanceulen.

Shamir, A. y Margalit, M. (2014). Technology and students with special educational needs: New opportunities and future directions. Routledge.

Unesco (2020). Interrupción educativa y respuesta al COVID-19. https://es.unesco.org/covid19/ educationresponse

Van Dijk, J. (2017). Digital divide: Impact of access. En P. Rössler, C. A. Hoffner y L. van Zoonen (Eds.), The international encyclopedia of media effects (pp. 1-11). John Wiley \& Sons.

Yaverbaum, G., Kulkarni, M. y Wood, C. (1997). Multimedia projection: An exploratory study of student perceptions regarding interest, organization, and clarity. Journal of Educational Multimedia and $\mathrm{Hy}$ permedia, 6(2), 139-153. https://www.learntechlib. org/primary/p/8907/

Yildiz, T. E. T. (2020). The use of ICTs in second language education: Opportunities and challenges. En O. Yildiz (Ed.), Recent developments in individual and organization adoption of ICTs (pp. 202-213). IGI Global.

Zheng, B., Warschauer, M., Lin, C, y Chang, C. (2016). Learning in one-to-one laptop environments: A Meta-analysis and research synthesis. Review of Educational Research, 86(4), 1052-1084. https://doi. org/10.3102/0034654316628645

Cómo citar este artículo: Echauri-Galván, B., García-Hernández, S. y Fernández-Gil, M. J. (2021). Enseñanza virtual de lengua inglesa durante el confinamiento domiciliario: percepciones y reacciones del alumnado en una universidad española. Íkala, Revista de Lenguaje y Cultura, 26(3), 603-621. https://doi.org/10.17533/udea.ikala.v26n3a08 
